\title{
Research on Community Based Aged-Care at Home Information Services in Cloud Computing Environment
}

\author{
Li Xiaoqin \& Wang Jing \\ School of law, Jiangxi Science and technology normal university, Nanchang, 330013, China
}

\begin{abstract}
The rapid increasing of aging population is becoming a serious challenge and how to improve the aged-care industry to obtain suitable services for the old is a burning issue in our society. Cloud computing provides low cost, high scalability, availability and disaster recoverability which can be a natural solution for some of the problems faced in aged care information services. After examining the main problems existing in current aged-care at home service and analyzing the advantages of cloud computing, the paper systematically studies the influence of community based aged-care at home information services in cloud computing. The studies show that the powerful ability of data storages and credible security of cloud computing will provide an information services platform, which can update resources at any time for aged-care and will cut the cost of information acquisition of users and enlarge the information services of aged-care.
\end{abstract}

KEYWORD: aged-care service; information service; cloud computing

\section{INTRODUCTION}

The world's population is ageing rapidly and the cost of caring for older people is also rising. For example in $2012,6.9 \%$ of the world population were more than 65 years old, and this is estimated to increase to around $20 \%$ by 2050 (Donald Kerr 2013). In China, according to the sixth national census in 2010 statistics, $13.26 \%$ of the population was over the age of 60 years which is increased by $2.93 \%$ compare to the national census in 2000. In the same time it is expected that we will face a tremendous shortage of qualified professionals in the aged care sector (Mathews 2008). This has brought enormous challenges to the regions and social economic development. To overcome these problems, many strategies has been proposed by governments worldwide to build more perfect social aged-care security system, aged society management system, aged life quality guarantee system and the industry for the elderly. In china, the traditional aged care mode is mainly by family care. But as the development of the economy and extending of the policy of family planning, the scale of family becomes smaller. In this context, the aged care service supported by family will gradually decrease while increase by society, so carrying out aged care at home with community service is the best aged care mode in china on combining the advantages of family care and social care(Liu Mancheng 2012).
But as the requirements of the aged become diversifying and complicating while the aging trend is becoming worsen, the information of homely aged care is taken on an explosive growth. This results in directly various huge resources requirement, time consumption and in the end influence the existence and development to the current aged care mode. Therefore, how to improve the aged-care industry to obtain suitable services for the old is a burning issue in our society.

Cloud computing is a new large distributed computing paradigm which can provide low cost, high scalability, availability and disaster recoverability which can be a natural solution for some of the problems faced in aged care information services. Its kernel is the sharing of network resources and the integrating of massive information. It can solve the massive information processing problem in low cost. These distinct advantages are just in line with the needs of aged care informationization, so in this paper, after examining the main problems existing in current aged-care at home service and analyzing the advantages of cloud computing, the paper systematically studies the influence of community based aged-care at home information services in cloud computing.

The rest of this paper is organized as follows. In Section II, we introduce the fundamental of the cloud computing in section II and detail the advantages of cloud computing based aged-care 
information services in section III. In section IV, we analyze the requirements of an aged-care at home information service in cloud computing environment and in section $\mathrm{V}$, we give the conclusions to our works.

\section{CLOUD COMPUTING}

Cloud computing is evolved from distributed computing, parallel computing and grid computing. It is a super computing paradigm that based on internet and it can integrate large amount of hardware and software resources and applications that are distributed among various servers, personal computers, mobile phones and other devices to work cooperatively.

\subsection{Principles of cloud computing}

Cloud computing is a general term for anything that involves delivering hosted services over the Internet. From the techniques point of view, cloud computing has experienced distributed computing, parallel computing, Software as a Service(SAAS) four development stages and public SAAS can be regarded as a earlier services provided by cloud computing. Basically, cloud computing has the following so called "5-3-2 principle" refers to the five essential characteristics of cloud computing, the three cloud service delivery methods, and the two deployment models which when put together properly describes what cloud computing is(Ambrust 2010, Jadeja 2012).

First up is he "5 Essential Characteristics of Cloud Computing": On-demand and self-service, ubiquitous network access, location transparent resource pooling, rapid elasticity, and measured service with pay per use. The characteristics were defined by the National Institute of Standards and technology (NIST) as part of their "Definition of Cloud Computing" publication, and they mostly speak for themselves as far as what each means at first glance. This publication is also where the principle is derived from. These five characteristics are all required for something to be qualified as cloud computing, according to NIST.

The 3 stands for the three service delivery methods, namely: Software as a Service (SaaS), Platform as a Service (PaaS), and Infrastructure (IaaS). All services being offered using cloud computing would fall under one or more of the above delivery methods, whether it be office applications and games (SaaS) or cloud backup storage or computing resources (IaaS). It becomes easier to define whether the service you are using is really of cloud computing or not just by judging if it is being delivered through one of the above methods.
And lastly, the 2 stands for the two main deployment models of cloud computing: the private and the public cloud models. NIST lists down four deployment models, but the other two is really just either a combination or a derivative of these two main ones. The public cloud is meant for public consumption while a private cloud's infrastructure is dedicated for private use, like in large corporations or government agencies.

\subsection{The value and advantages of cloud computing}

The essential spirits of internet is freedom, equality and sharing. So cloud computing is a computing paradigm that can best accord with the spirits of internet for its security, convenience, data sharing and limitless possibility characteristics, and it will change our works and lives from various aspects based on these values and advantages(Beamish 2008, Chappell 2007).

(1) Security. Cloud computing can provide reliable and security data storage center which can help the user to prevent the problem of data loss and virus intrusion. If we store the data in our personal computers, we often will suffer from the data damage for our own mistakes of virus intrusion. On the contrary, if we store our documents in the "cloud", we will not face such annoyance, for in the other end of "cloud" there is a professional technique team to manage the data storage, and at the same time, the strict privilege management policies can make us sharing our data with appointed people freely.

(2) Convenience. The user side low equipment requirement of cloud computing make it very convenient to use and it is no need to update the user' operating system and various kinds of user applications, as well as no need to update user's computer hardware. A client can share the "cloud" services as long as he or she has a computer with browser and can browse the internet, such as directly edit a document in the "cloud" side in the browser or share some information with friends.

(3) Data Sharing. Cloud computing can easily realize the sharing of data or applications among different devices. In the network application mode in cloud computing, the data is only stored one time and one place that is the cloud, and all the electronic devices such as PC,PDA and so on, can access and use the same copy of the data simultaneously if only the devices are connected with internet, this makes all the things simpler.

(4) Limitless possibility. Cloud computing provide almost limitless possibility to use the network for the client, such as it provides almost infinite storage space and computing power which is impossible in a personal computer. But in the cloud side, the huge clusters which are composed of millions and thousands servers can easily realize that. 
In additionally, individual or single device usually has limited computation capability while the potentiality of cloud computing is nearly limitless.

\section{ADVANTAGES OF CLOUD COMPUTING AGED-CARE INFORMATION SERVICE}

The use of cloud computing techniques in homely aged care has many advantages:

(1) Cloud computing provides a ubiquitous platform for the aged-care information services. In cloud computing mode, the data of client is stored in the back end servers. Clients can access the data via any computer or terminal and use the applications via network. Any one who has the appropriate internet connection and a standard browser can access the "cloud". The simplest cloud computing application can be found anywhere nowadays, such as the search engine, email and so on. In the early cloud computing product of Google search engine, the search requirement is sent to the large server clusters via internet and the search results return to the desktop of users. This ubiquitous distribution and society features provides a super power storage and computing capability platform to serve the agecare information services and help the clients to extract the valuable information from the massive data quickly. Additionally, cloud computing providers have professional technicians who can provide various kinds of security detection and security safeguard of anti-virus or Trojan horse. In a word, we can say that the cloud computing provides the aged-care information service a back end guarantee with data security and technique supports, at the same time provides a ubiquitous front end with convenient and rapid service(Ambrust 2010, Perkins 2007).

(2) Cloud computing will lower the cost of homely aged care by its scale effects. Since one of the main situation we are facing now in homely aged care is that the information and data of homely aged care are growing explosively, so how to integrate and sharing information becomes a key problem. While it is the kernel of cloud computing. Therefore, introducing the cloud computing into aged care to collect all the aged and their relative information then effectively categorize and integrate it into a cloud system will largely lower the cost of homely aged care.

(3) Cloud computing will help the intelligent configuration of aged care resources. By using clouding computing techniques to configure the resources intelligently, an wholly automatic homely aged care model can be built. This will certainly reduce the demand of manpower service. At the same time, because the easy use of cloud computing, it is no need the user to deeply master or to be an expert of it, this will significantly reduce the demand of the professional cloud computing human resources.

(4) Cloud computing will provide intelligent and integrated aged care services. The aged care model will take their full advantages of the investment by government and all sectors of society to satisfy the requirement of the aged while meet the expectations of government and various social welfare agencies. This type of aged care service mode will also enhance the standardization and transparentizing of aged care service as well as beneficial to the participation degree and the socialization degree of homely aged care.

(5) Cloud computing will increase the resources using rate of aged-care information service. The information in the "cloud" can be update at any time. So the client can get the newest information service at anytime and can running the various kinds of existed resources and computation capacity, this will simplify the complex procedure of information query, acquisition, organization. The client need only a "cloud" side terminal to get the information or services easily. In addition, cloud computing can $t$ share the aged-care information resources to the utmost extent to make the distribution and using of the resources more reasonably and more fully, and in the end increase the resource using rate and social values (Beddy 2012, Donald Kerr 2013).

\section{CONCLUSION}

The rapid increasing of aging population is becoming a serious challenge. Cloud computing provides a natural solution for aged-care information services. After examining the main problems existing in current aged-care at home service and analyzing the advantages of cloud computing, the paper systematically studies the influence of community based aged-care at home information services in cloud computing. Owing to the powerful ability of data storages and credible security of cloud computing will provide an information services platform, which can update resources at any time for aged-care and will cut the cost of information acquisition of users and enlarge the information services of aged-care.

\section{ACKNOWLEDGEMENTS}

The authors wish to thank the science and technology project of the education department of Jiangxi province under the grant GJJ14582 and thank the authors of the literature that we use in this article. 


\section{REFERENCES}

[1] Ambrust, M. et al.. 2010. A View of Cloud Computing. Communications of the ACM, 53 (4):5058.

[2] B.Eswara Beddy, T.V.Suresh Kunmar, Gandikota Ramu. 2012. An Efficient Cloud Framework for Health Care Monitoring System. International Conference on Cloud and Services Computing, 11;113-117.

[3] Dalia Sobhy, Yasser El-Sonbaty, Mohamad Abou Elnasr. 2012. MedCloud: Healthcare Cloud Computing System. The $7^{\text {th }}$ International Conference for Internet Technology and Secured Transactions, 7: 161-166.

[4] K.M.Yashpalsinh Jadeja. 2012. Cloud computing concepts, architecture and challenges. International Conference on Computing, Electronics and Electrical Technologies, 9: 877-880.

[5] Liu Man-cheng,Zuo Meiyung,Li qiu-di. 2012. Research on Informatization Demand for Aged-care at Home on the
Basis of Community Service. China Journal of Information Systems, 11: 87-100.

[6] M.Ahn, J.O.Beamish, R.C.Goss. 2008. Understanding Older Adults' Attitudes and Adoption of Residential Technologies. Fam. Consum. Sci. Res. J., 36: 243-260.

[7] N.L.Chappell. 2007. Handbook of Aging and the Social Science ( $3^{\text {rd }}$ Ed.). New York, Academic Press, 7:211: 223.

[8] Peeters,J.M., de Veer,A.J.E., Van der Hoek,L.,Francke,A.L.. 2012. J. Clin. Nurs. 21,3183-3193.

[9] Sum, S., Mathews, R.M., Hughes, I., Campbell.2008. Cyberpsychol. Behav. 11: 208-211.

[10] S. V. Donald Kerr, A.T. Khoei,A.H. Ghapanchi. 2013. Analysis of Research in Adoption of Assistive Technologies for Aged Care. $24^{\text {th }}$ Australasian Conference on Information Systems, 12: 4-6.

[11] Y.Li, A.Perkins. 2007. The impact of technological developments on the daily life of the elderly. Technol. Soc., 29:361-368. 